\title{
GÉNERO, EDUCACIÓN Y UNIVERSIDAD. UN ACERCAMIENTO \\ A LA HISTORIOGRAFÍA DE MÉXICO
}

\author{
Maestra Elva Rivera Gómez \\ Profesora e Investigadora \\ Centro de Estudios de Género \\ Benemérita Universidad Autónoma de Puebla \\ México
}

\begin{abstract}
RESUMEN
El trabajo presenta un balance historiográfico sobre el estado que guardan los estudios sobre las académicas e investigadoras de las universidades públicas de México en los últimos veinticinco años. Presentamos las etapas en que estas investigaciones se han realizado, las universidades en donde este tema ha sido objeto de estudio por las académicas y en las que el proceso de institucionalización de los estudios sobre las mujeres han sido impulsados por las feministas de diversas corrientes. También se incluyen los estudios realizados en tres universidades: la Universidad Nacional Autónoma de México, la Universidad de Colima y la Benemérita Universidad Autónoma de Puebla.
\end{abstract}

Descriptores: Género. Educación superior. Mujeres. Feminismo. Historiografía. México, Colima, Puebla. Siglo XX.

\section{INTRODUCCIÓN}

El estudio sobre las mujeres, en particular en la educación superior, nos lleva a señalar algunos aspectos: las actitudes culturales de la sociedad hacia las mujeres, poca participación en la toma de decisiones, la reproducción de estereotipos socioculturales en el quehacer universitario, por una parte, por la otra estudiar el proceso de incorporación 
femenina a la docencia e investigación universitaria, explicar cómo se ha llevado éste y cuales han sido los obstáculos y logros que han tenido que enfrentar las académicas al interior de las universidades.

De ahí, que este trabajo presenta un balance historiográfico sobre el estado que guardan las investigaciones dedicadas al estudio sobre la participación de las académicas e investigadoras en instituciones públicas de educación superior en México en las últimos veinticinco años. Partimos de algunas interrogantes: ¿Por qué nuestra cultura y sociedad, durante mucho tiempo, ha dado prioridad a la educación doméstica y no a la escolar de las mujeres?, ¿Por qué pocas mujeres tuvieron el privilegio de tener acceso a la educación superior hace unas décadas?, ¿Por qué en los últimos años se ha incrementado el porcentaje de mujeres en la docencia universitaria? Son preguntas que los estudios sobre las mujeres y/o de género intentan responder. Por supuesto que las fuentes existentes en cada uno de los Archivos Universitarios, en los Estados, en la Secretaría de Educación Pública, en la Asociación Nacional de Universidades e Instituciones de Educación Superior (ANUIES), y del Consejo Nacional de Ciencia y Tecnología (CONACYT), etc., nos llevarán a conocer los antecedentes, así como el proceso de incorporación de las mujeres a la academia y a la investigación en cada una de las instituciones educativas del país.

Debo mencionar que las preguntas antes señaladas, me llevaron a la búsqueda de fuentes hemerográficas y bibliográficas de carácter nacional y regional sobre la temática. Aquí sólo presentamos un avance de la investigación "Género, educación y universidad: Las académicas de la Benemérita Universidad Autónoma de Puebla 1970-2000", referente a la historiografía, en la que destacan investigaciones, ponencias y artículos 
realizados por las académicas de las universidades: Nacional Autónoma de México, de Colima y la Autónoma de Puebla. Es importante destacar los aportes que han realizado las universitarias Latinoamericanas, al incorporar esta temática, desde un enfoque feminista, en las investigaciones de las ciencias sociales y las humanidades; contribuyendo así a hacer visible el papel de las académicas universitarias en los diversos países de la región.

Una categoría de análisis empleada en las investigaciones es la teoría feminista y la perspectiva de género, que permite entender que la educación de las mujeres ha sido construida a partir de estereotipos socioculturales, y que la historia e historiografía tradicional han privilegiado y reconocido la capacidad intelectual de los hombres sobre el de las mujeres; los estudios sobre las mujeres, en este sentido nos hablan de discriminación, sexismo en la ciencia y en la docencia universitaria. Las feministas y no feministas que han estudiado esta temática en las diversas disciplinas han contribuido a la deconstrucción del pensamiento androcéntrico en la academia y en la ciencia. Un gran aporte de las académicas universitarias en el caso de México, ha sido la creación de la

Red Nacional de Centros, Programas de Estudios de la Mujer y/o de Género que incluye aproximadamente a más de 30 universidades Públicas y Privadas, desde dónde se ha discutido la importancia de los estudios de las mujeres en las universidades.

\section{1. ¿QUÉ IMPORTANCIA TIENEN LOS ESTUDIOS FEMINISTAS SOBRE}

\section{LAS MUJERES Y/O DE GÉNERO EN LAS UNIVERSIDADES?}


Uno de los debates llevados a la academia por las universitarias feministas en México y en Latinoamérica ha sido y es ¿Qué importancia tienen los estudios feministas, sobre las mujeres y/o de género en las universidades?, discusión llevada a cabo en el Primer Coloquio Nacional de Centros y Estudios de Género de las universidades nacionales, celebrado en Chapala, Jalisco en 1999, el otro efectuado en la Antigua, Guatemala en el 2001, en el marco del Primer Encuentro Mesoamericano de Estudios de Género, en donde se planteó la pregunta: ¿Pueden los estudios de género entrar a la Universidad? La respuesta de la gran mayoría de las académicas fue: no sólo pueden, sino deben estar en las universidades y los más recientes llevados a cabo en México: el Seminario: Feminismos Latinoamericanos, organizado por el Programa Universitario de Estudios de Género de la UNAM (abril, 2002), el cual logró reunir a las pioneras del feminismo Latinoamericano, a las académicas y a las activistas en sus diversas corrientes y el VI Encuentro de Centros y Programas de Estudios de la Mujer y de Género en Instituciones de Educación Superior en América Latina y el Caribe (octubre, 2003).

Para el caso Latinoamericano, es importante destacar el trabajo realizado por Gloria Bonder, en la obra titulada Estudios de la Mujer en América Latina, obra colectiva en el que se presenta un recorrido histórico por los países de Brasil, Chile, Colombia, Costa Rica, México, Paraguay, Perú, Puerto Rico, Republica Dominicana, Uruguay y Venezuela, escrito por diversas protagonistas (pioneras del feminismo en las universidades), en él las autoras analizan los procesos en que surgen los estudios de la mujer en estos países. A través de estos trabajos podemos conocer cuáles han sido las etapas, obstáculos y alcances del movimiento amplio de mujeres y de los estudios en cada 
una de las regiones de América Latina, para dar paso a la apertura de los espacios académicos.

El movimiento de las mujeres, señala Bonder, dejó como saldo una masiva incorporación de las mujeres a la educación media y superior en la región latinoamericana durante la década de los setenta. Este proceso de expansión cualitativa tuvo como efecto modificar las expectativas femeninas, quienes al elevar su nivel educativo desarrollaron aspiraciones de participación y autonomía.

Los años ochenta se caracterizaron por el retorno a la democracia en los países de la región. El clima de debate y apertura propició que mujeres activistas e investigadoras iniciaran la crítica en las distintas disciplinas científicas, con el fin de demostrar sus sesgos sexistas y demandar la democratización de las relaciones entre hombres y mujeres en el ámbito del hogar y de las instituciones sociales y políticas.

Bajo estos ejes se inician los procesos de institucionalización de los Estudios de la Mujer en los países de la región. La necesidad de integración de los estudios de la mujer y/o de género en las universidades aparece en todos los trabajos como iniciativas individuales o de pequeños grupos, destaca Bonder. Lo interesante de esta integración es que siempre se inicia en las universidades públicas. Una de las prioridades será lograr que en las universidades se integren institucionalmente los Estudios de la Mujer como política para la Educación Superior. Así, el panorama latinoamericano refleja una problemática común de las mujeres en las universidades.

Esto significa que los aportes teóricos de las académicas universitarias al pensamiento científico social han sido importantes. En México los primeros trabajos que se realizaron datan de la década de los ochenta del siglo XX. Las investigaciones 
realizadas a partir de este tiempo, nos plantean diversos enfoques: sociológicos, educactivos, antropológicos y muy pocos dedicados al estudio histórico. Es importante señalar que falta realizar una revisión historiográfica más amplia, que permita identificar qué temáticas se han investigado y cuáles faltan por realizar. He seleccionado para este trabajo sólo aquellas fuentes que, desde mi punto de vista, pasarán a formar parte de la historiografía de las mujeres y la educación superior en el país.

En México, la creación de seminarios, áreas, programas y centros de estudios de la mujer y/o de género, a iniciativa de las feministas mexicanas de los años setenta, ha rendido su primeros frutos, pues desde estos espacios no sólo se han legitimado estos estudios en la academia y en las universidades, sino también se han impulsado las investigaciones sobre las diversas problemáticas que atañen a las mujeres en la educación desde una diversidad de enfoques, incluyendo el feminismo. Para conocer estos debates, el trabajo escrito por la feminista mexicana Eli Bartra, Estudios de la mujer. ¿Un paso adelante, dos pasos atrás?, plantea los vínculos y la distancia entre el feminismo y los estudios de la mujer. Bartra presenta el desarrollo de estos estudios en la educación superior en México, en particular su significado, sus contradicciones y las barreras que enfrentan. De los puntos medulares en el debate feminista al interior de la academia se ubican la distinción entre estudios de la mujer y los de género, la burocratización de los programas, su institucionalización y evaluación, lo cual podría llegar a poner en riesgo su espontaneidad y creatividad.

Por su parte, Blanca Elvira Villarreal, en el trabajo Modelo de análisis de las trayectorias de formación de las investigadoras, señala que los cambios han modificado los espacios sociales de las mujeres contemporáneas y afirma que la perspectiva de 
género permite conocer esos cambios. Uno de éstos, es el universitario, el cual no deja de estar influido por las relaciones de género en aspectos relacionados con el status académico, el desempeño en el trabajo y la presencia en los órganos de gobierno:

\section{a) Estatus académico}

Las mujeres tienen un rango académico inferior, aun cuando las calificaciones de un doctorado y de productividad sean constantes. La investigación de esta autora demuestran que los efectos acumulativos de discriminación contra las mujeres en su carrera es perpetuado y magnificado. El número, cada vez más grande, de mujeres en bajas posiciones ha provocado el reclamo de éstas por su condición de temporales (de tiempo parcial o medio tiempo) y de asistentes durante muchos años para que se considere seriamente su definitividad o promoción.

\section{b) Desempeño en el trabajo académico}

Para las mujeres que se encuentran en posiciones inferiores o de asistentes no es fácil la publicación de sus trabajos, a pesar de que tienen que demostrar mayor productividad que los hombres. La participación en conferencias o congresos permite compartir resultados de investigaciones, debatir intelectualmente y de esta manera facilitar la posterior publicación de trabajos, sin embargo, hay datos según Villarreal, que señalan que las mujeres asistieron y organizaron menos conferencias que los hombres. Las mujeres con hijos buscan una productividad, tal vez para contrarrestar el estereotipo de ama de casa y de madre abnegada. También por la discriminación que hay en el ámbito laboral las mujeres reaccionan siendo más competitivas. 


\section{c) Participación en órganos de gobierno e instancias administrativas}

Las mujeres tienen un déficit tangible en su vida laboral causado por la exclusión o colaboración con los hombres. No es de sorprender que las mujeres tiene menor poder e influencia en sus departamentos que los hombres. Algunos indicadores muestran que ellas tienen menor probabilidad de tomar el rol activo en las reuniones, también que tienen una menor proporción y representación en puestos de toma de decisiones. Alguna mujeres dicen no tener iniciativa para obtener puestos de influencia, sin embargo, otras reconocen que existen barreras para lograr puestos importantes y que para lograrlo el esfuerzo tiene que ser mayor. De ahí que las redes de mujeres sirvan para muchas funciones, como para promoverse, dar asistencia profesional, incrementar la influencia política, dar apoyo emocional y social.

Aunque formalmente en las universidades no existe la discriminación debido a la igualdad jurídica (derechos y obligaciones), en los hechos, en la vida personal de las mujeres investigadoras salta la doble jornada femenina, ya que con muchas dificultades logra conciliar la vida familiar y la vida profesional plantea Mercedes Carreras, que en 1985 realizó el estudio acerca del El personal femenino en la UNAM; este trabajo, puede considerarse cómo uno de los pioneros dedicados a las mujeres universitarias. En el que señala que los factores que podrían interpretarse como discriminatorios en el designio académico laborales de las mujeres de la UNAM, se encuentran en esferas que se interrelacionan: Familiar. Espacio de socialización en cuanto a incentivos culturales y educativos; Cotidiana. La organización de su vida diaria en función del nivel de

compromisos familiares y sociales; Generacional. El momento que define la 
conceptualización del rol de la mujer y de las áreas propias para ella, así como las características propias del mercado laboral en que se insertan. Para la autora, la carrera académica de una mujer estará condicionada por los antecedentes socioeconómicos familiares, medidos por la escolaridad del padre y de la madre y el prestigio ocupacional del padre y/o madre. La esfera cotidiana, se explica sobre el argumento de la tensión entre la producción y la reproducción que forma parte también de una situación de la mujer cuando asume la profesión académica. Otros trabajos de Carreras publicados en 1989 titulados: "Las profesoras universitarias" y "Docencia Universitaria sobre la problemática femenina", en los que analiza la situación de las docentes universitarias a nivel nacional, en el que destaca la contratación laboral de las mujeres respecto a los docentes, así como también la diferencia salarial, los estudios de posgrado de las académicas.

Otra investigación relacionada con nuestro tema es el de Gabriela Delgado Ballesteros, académica de la UNAM, titulado "Resignificando la condición de las mujeres académicas de la Universidad Autónoma de México”. Estudio realizado desde la perspectiva de género en el que la autora destaca el tipo de contratación de las académicas universitarias en 1995:

"...del total de académicas en la UNAM, el 50\% estaban contratadas por horas asignaturas, el $30 \%$ de medio tiempo y el $20 \%$ de tiempo completo... El porcentaje de varones con definitividad es mayor que el de mujeres, solamente en los medios tiempos son equivalentes y en el caso de contrataciones por hora, son más mujeres..." (Delgado 2001: 61-74).

Con respecto a las condiciones en que desempeñan su quehacer académico, la autora encuentra que la productividad académica de las mujeres está estrechamente 
vinculada a dos cosas: el combinar el espacio laboral con la maternidad y los estudios de especialización. Según las entrevistas realizadas a las académicas, Delgado llegó a la conclusión que:

"...La productividad académica se logra dependiendo del ciclo de vida o después del maternaje. Estos aspectos se evidenciaron claramente con el análisis de la currícula, al encontrar espacios de baja productividad, tanto en las horas frente a grupo como en la publicación de artículos, manuales o libros. El dedicarse al maternaje impide un desarrollo académico continuo, existen espacios temporales de logros de actualización o grados académicos dependiendo de la época de la maternidad y el maternazgo..." (Delgado 2001: 61-74).

Norma Blázquez Graff, fue integrante del Grupo por la Mujer en la Ciencia en 1984. A partir de esa fecha ha publicado diversos artículos sobre las mujeres en la ciencia, entre los que podemos mencionar los siguientes: "La mujer en la ciencia" (1984); "Incorporación de la mujer a la ciencia a comienzos de los noventa", (1987); "Relaciones entre la mujer y la ciencia" (1989); "Participación de las científicas en México" (1989) y “Acerca de las científicas de la UNAM” (1990). En estos trabajos la autora destaca los avances que han tenido las mujeres en la ciencia, el lugar que ocupan en el Sistema Nacional de Investigadores en cada una de las áreas de conocimiento. Así como también señala la discriminación que han sido objeto las mujeres científicas en las evaluaciones para escalar niveles en el Sistema Nacional de Investigadores y para obtener recursos para desarrollar proyectos de investigación relacionados con los temas de las mujeres, los cuales son financiados por el Consejo Nacional de Ciencia y Tecnología.

Olga Bustos en el trabajo titulado "Los estudios de la mujer (y de género) en la UNAM: investigaciones y tesis", presenta un balance sobre las diversas investigaciones que se han realizado en la Universidad Nacional Autónoma de México en las últimas 
décadas y que fueron publicados en la obra colectiva Estudios de género y feminismo en 1989. Este trabajo es una referencia obligada de consulta para quienes deseen conocer el origen, avance, logros, enfoques y retos de los estudios de las mujeres realizados por investigadoras, académicas y estudiantes de esta universidad.

Nora Garro en el trabajo titulado "La mujer profesionista universitaria" (1989) y Liliana Morales Hernández en la investigación sobre "La mujer en la educación superior en México" (1989), presentan un análisis sobre las características de las profesionistas universitarias en el contexto nacional.

Hasta aquí hemos visto los estudios de carácter nacional y en particular los dedicados al análisis sobre la situación de las académicas e investigadoras de la UNAM. A continuación presentaremos las investigaciones realizadas por académicas de otras universidades del país.

Sara María G. Martínez, en el trabajo sobre "La participación de las mujeres en la academia. Condiciones actuales y retos" (2001), dedicado al análisis sobre la situación de las académicas de la Universidad de Colima, destaca que en esta institución en el rubro del posgrado, de todos los programas, en el de educación, las mujeres docentes ocupan el 47, 4\% con respecto a las otras áreas. En la investigación del Sistema Nacional de Investigadores, según registros, en esta institución académica, sólo el 20\% lo ocupan las mujeres. Como candidatas sólo se registran 4 mujeres que representan el 50\% de mujeres; en el nivel 1 se registran 5 mujeres que equivale al 16,6\% y en el nivel II de 6 integrantes no hay ninguna mujer. Por otra parte, en este artículo se destaca la situación de las mujeres en la dirección de las Universidades Públicas del país. Llama la atención que las mujeres que ocupan el cargo de rectoras sean únicamente el $2 \%$ y en cargos académicos 
de primer nivel apenas lleguen al $10 \%$ en las Universidades Públicas, el $4 \%$ en las Privadas y al 6\% en los Institutos Tecnológicos.

Otro estudio sobre las mujeres en la Universidad de Colima de Verónica Valenzuela, titulado "Perfil de las mujeres universitarias de Colima" (1995), el cual señala los grupos de edad de las mujeres académicas, la escolaridad de las madres de las académicas, áreas de preferencia de las mismas, así como la especialización de las docentes en el que distingue lo siguiente:

"...que la mayor parte de las mujeres universitarias de Colima se concentra en un rango de edad que va de los 24 a los 34 años. En esas edades se agrupa el 66.2\% de las que han cursado al menos una carrera profesional. Las mujeres que tienen actualmente entre 35 y 45 años son el 18\%. Esto significa que al igual que en el resto del país, la incorporación de la mujer a los niveles superiores de escolaridad no tiene más de cuatro décadas acelerándose su incorporación a partir de los años setenta.

La escolaridad de las madres, de universitarias es del $60 \%$ concerniente a educación a nivel primaria, sólo el $6,1 \%$ de las madres cuentan con una carrera universitaria y un $13 \%$ con el mismo nivel escolar.

Las áreas más socorridas por las mujeres en estudios superiores son las humanidades $(27,8 \%)$, ciencias de la salud $(22 \%)$ y ciencias sociales $(18,8 \%)$, siendo las carreras de pedagogía, enfermería y trabajo social las de mayor demanda - por el contrario en las áreas de ciencias naturales y ciencias exactas sólo se hallan un 2,6\% y en las disciplinas tecnológicas un $6,1 \%$.

La especialización de mujeres universitarias es muy bajo. El 86,1\% de las mujeres cuenta con tan sólo lo licenciatura, el 8,4\% ha estudiado una especialidad, el 4,3\% ha estudiado una maestría y el 0,9\% un doctorado..." (Valenzuela 1995: 40-47).

Florentina Preciado Cortés, dedica su estudio al análisis de la situación de las mujeres en la educación superior en la región occidente de la república mexicana que abarca los estados de Jalisco, Colima, Guerrero, Michoacán y Nayarit en el artículo "Lo público y lo privado en la educación femenina del occidente mexicano" (1997). Dicha investigación permite conocer que en la educación superior, según la autora, las 
exigencias sociales de épocas recientes han provocado una mayor participación de la mujer en las esferas académicas llegando casi a la igualdad con la matrícula masculina. Sin embargo, señala que, la distribución de áreas de estudio está tremendamente marcada por la diferencia de géneros.

“...La distribución por sexo presente en las áreas de conocimiento de la educación superior, de alguna manera se aprecian como extensión de lo doméstico, con cierta compatibilidad con el papel de esposa y de madre..." (Preciado 1997: 30).

Así, en la educación superior los conocimientos propios del género se agrupan en áreas como: ciencias sociales, humanidades y educación con lo femenino, mientras que las ingenierías y agropecuarias se relacionan con lo masculino. A esto hay que agregar el prestigio y reconocimiento del que gozan las segundas a diferencia de las primeras.

En este mismo estudio, la autora destaca la incorporación femenina en la Universidad y el papel de las estudiantes universitarias; en el primero señala algunas de las causas que originaron un incremento notable a la educación superior por parte de las mujeres:

“1) El desarrollo y el crecimiento económico del estado como parte de la modernización y la industrialización.

2) A partir de 1940 dentro de la teoría del desarrollo, la educación es considerada como "una inversión para la formación de recursos humanos". Esto justifica la capacidad de las instituciones para ubicar a la población dentro de la división del trabajo, de tal forma que el valor del mercado laboral encuentra correspondencia con el nivel de estudios.

3) A nivel nacional la autora distingue dos etapas de crecimiento de la educación universitaria. La primera corresponde al periodo de 1969 a 1977 y el segundo de 1977 a 1985. En 1969 la matrícula a nivel nacional en educación superior era de 186 041 alumnos, de los cuales 153935 (82.7\%) eran hombres y 32106 (17.3\%) mujeres; para 1977 la matrícula creció 1.89 veces, en el caso de los hombres a razón de 1.58 y la femenina al del 3.4. la distribución pasó al $73.7 \%$ y $27.3 \%$ respectivamente. 
Durante la segunda etapa, de 1977 a 1985, puede observarse la participación de la mujer de manera significativa, ya que el crecimiento de la matrícula a nivel nacional fue del 79\%; la matrícula masculina creció al ritmo del $59 \%$ y la femenina al del $135 \%$, tendiendo a su nivelación con el $65.6 \%$ y el $34.5 \%$ respectivamente.

4) Para 1990 la matrícula creció a 1078 191; de éstos 434803 pertenecían al sexo femenino y 643388 al sexo masculino..." (Preciado 1997: 30).

Como ya se mencionó anteriormente, la educación está estrechamente ligada al desarrollo del país, ciudad o región en la cual se encuentre. Así pues, señala Preciado Cortés que, los estados como Nuevo León, Sonora y el Distrito Federal presentan los porcentajes más altos en asistencia escolar por ser éstos los estados más desarrollados; en contraparte estados como Chiapas, Guerrero y Michoacán son los que presentan menor asistencia escolar por ser estados poco desarrollados.

El segundo punto se refiere a las universitarias, el estudio distingue que en las áreas universitarias están muy marcadas las tendencias académicas en función del género. Así, en 1994 se realizó una encuesta en la que se observan las tres áreas de mayor concentración femenina la cuales son: Educación y humanidades, ciencias de la salud y ciencias sociales y administrativas. Cubriendo el $65,2 \%, 59,4 \%$ y 54,3\% respectivamente.

Por otro lado, la mayor concentración masculina se encontraba en las ciencias agropecuarias con un 79,1\%, ingeniería y tecnología con un 74,5\%. Esta situación se refleja en el campo laboral, donde las carreras "masculinas" destacan con más de diez ingresos mínimos, mientras que las carreras "femeninas" son las de menor salario. Si bien, por causas ya mencionadas anteriormente, la matrícula femenina casi se ha igualado con la matrícula masculina universitaria, en el campo laboral es algo que, lamentablemente, no se ve reflejado en esta zona (occidente); según estadísticas, de los 
235856 profesionistas que se encuentran, el 67\% son hombres mientras que sólo el 33\% son mujeres.

“...Quizá la educación no ha dejado de ser concebida como una antesala al matrimonio, las expectativas no logren rebasar el ámbito de lo doméstico, o es el mismo medio ambiente y las condiciones laborales y sociales las que impiden que se incorporen a la población económicamente activa..." (Preciado 1997: 36)

Para el caso concreto de la incorporación de las mujeres a la universidad de Colima, Preciado señala que:

“...La universidad de Colima, en particular, presenta un ritmo de crecimiento mayor en la última década. Así tenemos que en 1980 había 476 mujeres inscritas y para 1992 ascendió el número a 2 553, lo que refleja un incremento de 436\%, mientras que el incremento de la matrícula masculina fue del 105\%: de 1317 inscritos en 1980 pasó a 2710 en 1992. Para 1994 la población inscrita en el nivel superior dentro del estado se encontraba de la siguiente manera: 3463 mujeres y 3 854 hombres, cantidades que equivalen al $47.4 \%$ y $52.6 \%$ respectivamente..." (Preciado: 1997: 33)

Como los demuestran estas investigaciones, en las universidades se reproducen los estereotipos socioculturales sexistas que impiden mejorar la productividad y rendimiento de las académicas, investigadoras y estudiantes. Ya que nuestro tema central, es el caso poblano, a continuación presentamos el origen de los estudios de la mujer en la academia y las investigaciones que sobre esta temática se han realizado.

\section{LOS ESTUDIOS SOBRE LAS MUJERES Y DE GÉNERO EN LA BUAP}

En el caso de la Benemérita Universidad Autónoma de Puebla, el interés por investigar los temas sobre las mujeres, surge en la década de los 70, en una etapa de la 
historia de la universidad que corresponde a la Universidad Crítica, Democrática y Popular. Al frente de la rectoría se encontraba el Ing. Luis Rivera Terrazas, predominaba al interior el Partido Comunista Mexicano. El ambiente político posibilitó la llegada de las ideas feministas a la academia, en particular, la incorporación de académicas exiliadas sudamericanas, de académicas militantes de la izquierda de otras universidades como: la de Guerrero, Sinaloa y la UNAM; así como también de las jóvenes militantes comunistas y de otras fuerzas de izquierda. Todas ellas incorporadas al estudio, a la docencia o al trabajo administrativo en las diferentes escuelas y dependencias de la institución. Sin lugar a duda, la participación de ellas en la vida académica y política posibilitó ampliar su radio de acción al interior de los sindicatos universitarios, a través de las Secretarías de Asuntos Femeniles. Desde esta secretaría fue donde se elaboraron diversas iniciativas en pro de las académicas y trabajadoras, las cuales fueron reconocidas e incorporadas en las cláusulas del contrato colectivo de trabajo: las prestaciones sociales, el servicio del Circulo Infantil (estancia infantil), etc.

En marzo de 1980, un grupo de académicas sindicalistas, a través de la Secretaría de Asuntos Femeniles de la sección 15 del Sindicato Único Nacional de Trabajadores Universitario (SUNTU), a cargo de Clara Angélica Ureta Calderón, organizaron el Primer Encuentro Sindical sobre la Condición de la Mujer. En el que participaron Nicole Vaisse, Amalia García, María Teresa O’Connor, Marcela Lagarde, Alaíde Foppa, entre otras. Los resultados del encuentro fueron publicados ese mismo año y en él encontramos una diversidad de temas como: la teoría feminista, mujer y trabajo, maternidad voluntaria, participación política de la mujer en los partidos políticos, e incluye un trabajo 
que puede considerarse como el pionero sobre la situación de las académicas sindicalistas de la Universidad Autónoma de Puebla.

Desde esta secretaría, algunas académicas participaron en diversos foros nacionales convocados por el movimiento feminista, por el movimiento autónomo de mujeres y por las militantes de los partidos políticos de la izquierda mexicana.

En 1980, se creó el Colegio de Antropología de la Escuela de Filosofía y Letras. Marcela Lagarde funda el Taller de Antropología de la Mujer, al que asistían principalmente estudiantes del colegio y universitarias de otras disciplinas. En 1981 organiza el encuentro: La Mujer y la Antropología Mexicana. El 9 y 10 de marzo de 1982, lleva a cabo el Foro Internacional de la mujer "Alaíde Foppa”, en el que participaron representantes de Guatemala, El Salvador, Chile, Uruguay, Argentina, Nicaragua, Haití y México. En este foro se presentaron dos trabajos sobre las mujeres de la BUAP, elaborados por las estudiantes de Antropología Martha Silvia Reyna Pacheco y Antonella Fagetti. La primera presentó el estudio comparativo sobre la situación de algunas mujeres trabajadoras, -que además eran estudiantes- y de académicas de la UAP; en tanto la segunda analizó el tema de la maternidad y trabajo, ambas realizaron entrevistas a mujeres y hombres y coincidieron en señalar que las universitarias en estos años no estaban exentas de las condiciones de opresión, independientemente de su condición de trabajadoras administrativas o académicas; así también distinguen las condiciones y prestaciones laborales de las mujeres universitarias, como son: las prestaciones (jubilación, servicio del Circulo Infantil, permisos para estudiar), las jornadas laborales, aunado a éstas las de carácter familiar, mostrando así una 
aproximación de la situación de las académicas a principios de los años ochenta (Universidad 1982: 12-15).

Durante este rectorado también es importante señalar, que a través de la Dirección de Extensión Universitaria, el Taller de Antropología de la Mujer y la Secretaría de Asuntos Femeniles del SUNTUAP en la UAP, se organizaron conjuntamente diversos eventos conmemorativos al 8 de marzo "Día Internacional de la Mujer”, durante los años de 1982 a 1985. Años mas tarde el Taller de Antropología de la Mujer, se transformaría en Seminario en Estudios de la Mujer.

En los años 90, bajo la rectoría del Lic. José Doger Corte, la Universidad lleva a cabo una reforma profunda: se inicia paulatinamente la reducción de la matrícula universitaria, del personal docente y administrativo, se implanta el sistema de créditos, se lleva a cabo la homologación del personal académico, se inicia con los sistemas de evaluación al personal docente. En este contexto los planes de estudio de las licenciaturas se transforman, el seminario de estudios de la mujer Colegio de Antropología se transformaría en estudios de género (1994) y -en la última transformación del plan de estudios- éste se denomina género y sexualidad (2002).

Por otra parte, en 1995, María del Carmen García Aguilar funda el Centro de Estudios de Género, el cual es aprobado por el Consejo de Unidad de la Facultad de Filosofía y Letras de esta universidad, durante estos ocho años, éste centro ha realizado diversas actividades de investigación, docencia y extensión (García 2003).

Estudios particulares de las académicas de la Benemérita Universidad Autónoma de Puebla, se han realizado en diversas etapas y en su mayoría son estudios de casos 
analizados desde diversas disciplinas y enfoques feministas. En la cronología encontramos después de la década de los ochenta los siguientes trabajos:

En 1994 Silvia María Aguila Tecuatl, desarrollaba la investigación “Mujer en la academia: su experiencia profesional", investigación que no concluyó por su muerte. Gloria Tirado Villegas, ha realizado diversos trabajos sobre esta temática, entre los que destacan: "Si de mujeres se trata...Las universitarias de la BUAP" (Tirado 2001); "Las universitarias ante la evaluación académica" (2003a) y "Otra mirada al 68”. Mujeres en Puebla. (2003b). En el primer trabajo estudia los procesos de evaluación académica al personal docente y de investigación, señala que las mujeres competimos para tener acceso a las becas de toda índole, Desempeño Académico, para obtener recursos a los fondos de FOMES, para ser reconocidas y pertenecer al padrón de docentes con perfil PROMEP, etc. Todo esto, destaca la autora, nos ha llevado a una competencia con nosotras mismas y entre nosotras mismas, sobre todo a las casadas y madres solteras, ya que tenemos que combinar el espacio doméstico con el quehacer académico y además ser docentes de calidad y competir con nuestros colegas varones en desigualdad de condiciones. En el segundo trabajo aborda el tema de las condiciones académicas y socioculturales que enfrentan las docentes e investigadoras para concursar por las becas al desempeño académico, y así poder tener un ingreso extra en función a la productividad anual (horas de docencia, tutoría individual y colectiva, investigación, etc). El tercer trabajo lo dedica al estudio de la participación de las mujeres universitarias en el movimiento estudiantil de 1968 en Puebla, rescata las historias de vida de las principales dirigentes estudiantiles, haciendo visible el papel de las militantes comunistas y de las dirigentes estudiantiles y con ello contribuye a desmitificar los estudios sobre el 
movimiento estudiantil de 1968 en Puebla y da a conocer que muchas de estas mujeres, "invisibles" en la historia de la institución-, fueron maestras fundadoras de algunas preparatorias, representantes de órganos de gobierno y funcionarias de la institución en las décadas posteriores.

María del Rocío Andrade Santana y María de Jesús León Zermeño, en La organización del trabajo doméstico, en Maestros Universitarios, publicado en el año (2001), distinguen la distribución del trabajo doméstico en profesoras y profesores universitarios, señalando que el mayor porcentaje de horas de trabajo extraacadémico lo cubren las mujeres, destacan también los grupos de edad, las áreas de conocimiento en las que se ubican el profesorado entrevistado, grado académico, etc.

Podemos distinguir entonces, que de 1980 al año 2000, durante estos veinte años, en la universidad los estudios sobre las mujeres y de género han tenido sus primeros frutos al interior de la academia, ya que no sólo encontramos tesis de grado, sino también proyectos de servicio social e investigaciones en otras áreas del conocimiento; al exterior quienes investigan estos temas han establecido vínculos de cooperación con el sector gubernamental, no gubernamental y con organizaciones de mujeres. En la universidad y en la academia se transita de los estudios de las mujeres a los estudios de género. Así podemos afirmar, que la llegada del feminismo a la academia se debió a diversos factores: por la influencia de las militantes de la izquierda nacional y andina, por el impulso de académicas al interior del sindicalismo universitario, por la creación de seminarios de la mujer y de género en el Colegio de Antropología y del Centro de Estudios de Género en la Facultad de Filosofía y Letras de esta universidad. 
Por último, quisiera hacer referencia a los temas analizados en la Conferencia Mundial sobre la Educación Superior, convocado por la UNESCO, celebrado en Paris, en octubre de 1998. Uno de los debates temáticos de la Conferencia estuvo dedicado a las “Mujeres y educación superior: Cuestiones y perspectivas". Éste tuvo como objetivo examinar el estado actual de las principales cuestiones relacionadas con la situación de las mujeres en la educación superior. De manera particular se destacan los instrumentos internacionales que hacen referencia al acceso de las mujeres a la educación; en especial, la Declaración de Beijing de 1995. Según el Informe sobre Desarrollo Humano que publica anualmente el Programa de la Naciones Unidas para el Desarrollo:

- El 66\% de los analfabetos del mundo son mujeres;

- En los países en desarrollo, por cada 100 hombres presentes en el mercado de trabajo sólo hay 50 mujeres;

- Los PIB nacionales registrarían un aumento importante si el trabajo no pagado de las mujeres fuese un factor de la producción reconocido oficialmente.

- Las mujeres siguen padeciendo de la disparidad con respecto a los salarios de los hombres en múltiples sectores de la vida profesional (su salario es un $25 \%$ inferior, en promedio).

En este documento destacan tres aspectos con respecto a la Educación Superior y las mujeres.

1. En primer lugar, se debe considerar que las mujeres graduadas forman parte de la base de recursos humanos esenciales de sus respectivos países. Por eso, tienen derecho a las mismas oportunidades que sus colegas masculinos en lo referente al acceso a la enseñanza superior y a las carreras profesionales. 
2. En segundo lugar, habida cuenta de que la reforma de la educación superior es una prioridad, debería contraerse un firme compromiso de dotar a las mujeres con todas las competencias de gestión necesarias para contribuir a la renovación general de este sector de la educación.

3. En tercer lugar, la feminización de las funciones de dirección requiere ser analizada y definida con mayor claridad...”

De suma importancia para las mujeres, es el documento que hace referencia a Las mujeres en la Administración de la Enseñanza Superior, elaborado por la UNESCO en 1993, en el que se indican los principales obstáculos que impiden la participación de las mujeres en la esfera de adopción de decisiones:

$\checkmark \quad$ Acceso ilimitado a la educación en general, y a la educación superior en particular.

$\checkmark \quad$ Prácticas discriminatorias en materia de nombramientos y ascensos profesionales.

$\checkmark \quad$ Tensiones derivadas de la doble función familiar y profesional.

$\checkmark \quad$ Actitudes de la familia.

$\checkmark \quad$ Interrupciones de la carrera profesional.

$\checkmark \quad$ Estereotipos culturales.

$\checkmark \quad$ Alineación cultural masculina y resistencia continua a que las mujeres ocupen puestos de dirección.

$\checkmark \quad$ Propagación del "síndrome de la barrera", que da prioridad a los criterios encubiertos en materia de ascensos profesionales. 
$\checkmark \quad$ Ausencia de políticas y legislación adecuadas que garanticen la participación de las mujeres.

En el apartado VI de la Conferencia, se refiere a las mujeres en la dirección de la educación superior, así como también, se presentan en el apartado VII las estrategias para la actividad futura, en el que se señala que: “...en las universidades e instituciones de educación superior, deben crearse Oficinas para la Igualdad de Oportunidades en materia de Empleo, a fin de supervisar los progresos de las mujeres que ocupan puestos académicos y administrativos..."

Así el documento propone a los Estados miembros de la ONU, para el 2010, los siguientes objetivos:

- Los puestos de catedráticos, profesores y jefes de departamento, deben ser desempeñados por hombres y mujeres sobre un pie de igualdad. (50\% de los puestos)

- Lo ideal sería que el $50 \%$ de todos los puestos de rectores y vicerrectores fuesen ocupados por mujeres.

- El 50\% de los miembros de los órganos rectores de la educación superior deberían ser mujeres.

\section{CONCLUSIONES}

Como podemos ver a lo largo de este trabajo, las investigaciones sobre las mujeres y la educación superior, en nuestro país tienen aproximadamente unos treinta años de 
haberse iniciado. Sus raíces están relacionados con el movimiento popular de mujeres y con la llegada del feminismo a la academia.

Las autoras aquí analizadas coinciden que los factores de índole social, familiar y cultural han sido los principales indicadores de discriminación hacia las mujeres en las universidades. Corresponderá a las mujeres y los hombres eliminar esas barreras para incursionar a un mundo laboral, académico, de investigación, etc. en condiciones distintas a las que ahora tenemos ahora. Ésta no es una tarea fácil, de ahí que tenemos que emprender de manera conjunta, autoridades, personal académico, estudiantes, Estado y la sociedad en su conjunto las reformas necesarias para cambiar los escenarios docentes y de investigación.

Si bien es cierto que el progreso de una nación se mide por los indicadores educativos, el caso de nuestro país, según los informes internacionales, es reprobatorio. Acaso nuestros funcionarios y quienes diseñan las políticas educativas de este país se han dado cuenta que uno de esos indicadores negativos se debe a que a las mujeres no se les da la oportunidad de ingresar, permanecer y concluir los estudios básicos, y muchos menos tienen la oportunidad de ingresar a los estudios superiores y las que tienen ese privilegio lo hacen en desigualdad de condiciones; o que las mujeres que desempeñamos el ejercicio docente en las universidades tenemos que combinar doble o triple trabajo para demostrar nuestra eficiencia y capacidad académica para tener acceso a una categoría mayor, a becas, a puesto de dirección, cuando las condiciones laborales, económicas, sociales y culturales son discriminatorias para la gran mayoría de las mujeres.

Ante esta situación, considero que los estudios sobre la educación, en especial, los relacionados con el nivel superior, deben hacer visible el quehacer académico de las 
mujeres en la docencia, la investigación y en la toma de decisiones. ¿Cómo?, en primer lugar, que sus estadísticas no sólo estén desglosadas por sexo, sino también que se consideren otros indicadores; en segundo lugar, que se diseñen políticas educativas internas que lleven a potenciar el papel de las mujeres; en tercer lugar, que se impulsen los estudios sobre las mujeres en las propias instituciones: por facultades, departamentos, áreas, etc., así como también, que las universidades y autoridades permitan la inserción de cursos sobre estas temáticas y en cuarto lugar, que las instituciones destinen recursos para llevar a cabo este tipo de investigaciones al interior de las universidades. Todos estos elementos contribuirán sin lugar a dudas, a construir instituciones educativas a nivel superior más equitativas. Sólo de esta manera, el Estado y las universidades públicas conjuntamente con las académicas podamos cumplir con las expectativas trazadas por la UNESCO para el 2010; ¿no creen que podemos luchar por esto?

\section{BIBLIOGRAFÍA}

Acker, Sandra. 1994. Género y educación. Reflexiones sociológicas sobre las mujeres, la enseñanza y el feminismo. Madrid: Narcea.

Aguila Tecuatl, Silvia Ma. Rocio. 1994. "Mujer en la academia: su experiencia profesional." (Proyecto de investigación, inédito).

Andrade Santana, María del Rocío y León Zermeño María de Jesús. 2001. La organización del trabajo doméstico, en Maestros Universitarios. Puebla: COESPO.

Bartra, Eli. 2000. Estudios de la mujer. ¿Un paso adelante, dos pasos atrás?. México: Universidad Autónoma Metropolitana-Xochimilco.

Blázquez Graff, Norma. 1984. "La mujer en la ciencia." En Nueva Sociedad, (Cd. De México, México), num. 74: 141-145.

. 1989 b) "Participación de las científicas en México." En Memorias del

Primer Congreso Mexicano de Historia de la Ciencia y la Tecnología, T. 2. México: Sociedad Mexicana de Historia de la Ciencia y la Tecnología: 745-754. 
. 1989. "Relaciones entre la mujer y la ciencia." En Seminario sobre la participación de la mujer en la vida nacional. México: UNAM: 443-449.

. 1990. "Acerca de las científicas de la UNAM." En Congreso de la UNAM, foros locales, ponencia, febrero-marzo. México: Universidad Nacional Autónoma de México.

. 1997. "Incorporación de la mujer a la ciencia a comienzos de los noventa." En Tarrés, María Luisa, ed., La voluntad de ser. Mujeres en los noventa. México: Colegio de México.

Bustos, Olga. 1989.“Los estudios de la mujer (y de género) en la UNAM: investigaciones y tesis." En Estudios de género y feminismo, T. 1, México: Fontamara-Universidad Nacional Autónoma de México: pp. 123-147.

Carreras, Mercedes. 1985. "El personal académico femenino en la UNAM." En II Foro Universitario de la Mujer. México: Universidad Nacional Autónoma de México. . 1989a. "Las profesoras universitarias." En Seminario sobre la participación de la mujer en la vida nacional. México: Universidad Nacional Autónoma de México: 353-378.

1989b. Docencia Universitaria sobre la problemática femenina. Posibilidades y obstáculos. México: Universidad Nacional Autónoma de México.

Delgado Ballesteros, Gabriela. 2001. "Resignificando la condición de las mujeres académicas de la UNAM.” En Zapata Martelo, Emma, Vásquez García, Verónica y Alberti Manzanares, Pilar, coords., Género, feminismo y educación superior. Una visión internacional. México: Colegio de Posgraduados: 61-74.

García Aguilar, María del Carmen, Rivera Gómez, Elva y Montes Sosa, Gabriel. 2003. Los Estudios de Género en la Facultad de Filosofía y Letras: Ocho años de experiencia (1995-2003). Puebla, México: Benemérita Universidad Autónoma de Puebla.

Garro, Nora. 1989. "La mujer profesionista universitaria." En El Cotidiano, ( 27): 66-70. López Villareal, Blanca Elvira. 1997. "Modelo de análisis de las trayectorias de formación de investigadoras." En Hierro, Graciela, comp., Filosofía de la educación y género. México: Universidad Nacional Autónoma de México-Torres Asociados.

Martínez G., Sara María. 2001. "La participación de las mujeres en la academia. Condiciones actuales y retos." En GenEros. Revista del Centro de Estudios de Género de la Universidad Autónoma de Colima. (Colima, México),Junio de 2001, año 8, (24): 36-38.

Morales Hernández, Liliana. 1989. "La mujer en la educación superior en México.” En Universidad Futura, vol. 1, (1) México: Universidad Autónoma MetropolitanaAtzcapozalco: 68-77.

Preciado Cortés, Florentina. 1997. "Lo público y lo privado en la educación femenina del occidente mexicano." En GénEros, Revista del Centro de Estudios de Género de la Universidad de Colima. (Colima, México), Febrero de 1997, año 4, (11): 30-37.

Tirado Villegas, Gloria. 2001. "Si de mujeres se trata....Las mujeres en la BUAP." En Ureta Calderón, Clara, Lucero, Rocío y Rivera Gómez Elva, I Foro; las Mujeres en el nuevo milenio. México: Universidad Autónoma de Tlaxcala. 
. 2003a. "Las universitarias ante la evaluación académica." En Anuario del Seminario de Estudios Regionales del Departamento de Estudios Jurídicos de la Universidad de Guadalajara. Jalisco: Universidad de Guadalajara. . 2003b. "Otra mirada al 68". Mujeres en Puebla. Puebla: Benemérita Universidad Autónoma de Puebla. (en prensa).

UNESCO, 1998. Conferencia Mundial sobre educación Superior, debate temático: "Mujeres y educación superior: cuestiones y perspectivas". París: UNESCO.

Universidad. 1982. (Puebla, México), Año 2, (10 y 11). . 1984. (Puebla, México), Año IV, (8). 1985. (Puebla, México), Año V, (7).

Valenzuela, Verónica. 1995. "Perfil de las mujeres universitarias de Colima." En Géneros, Revista del Centro de Estudios de Género de la Universidad Autónoma de Colima. (Colima, México), Año 2, (6): 40-70. 